1 Universidade Regional do Cariri (Urca) - Crato (CE), Brasil.

geycyenf.ga@gmail.com

2 Faculdade de Juazeiro do Norte (FJN) - Juazeiro do Norte (CE), Brasil.

jeanderson17@ymail.com

3 Universidade Regional do Cariri (Urca) - Crato (CE), Brasil.

jam.ex@hotmail.com

4 Faculdade de Juazeiro do Norte (FJN) - Juazeiro do Norte (CE), Brasil.

cintiadelimagarcia@hotmail. com

\section{Violência psicológica em lésbicas, gays, bissexuais, travestis e transexuais no interior do Ceará, Brasil}

\author{
Psychological violence in lesbians, gays, bisexuals, transvestites and \\ transsexuals in Ceará, Brazil
}

Grayce Alencar Albuquerque ${ }^{\mathbf{1}}$, Jeanderson Soares Parente ${ }^{\mathbf{2}}$, Jameson Moreira Belém³, Cintia de Lima Garcia4

RESUMO Objetivou-se determinar o perfil de violência psicológica perpetrada contra Lésbicas, Gays, Bissexuais, Travestis e Transexuais (LGBTT) no interior cearense. Estudo quantitativo realizado com 316 indivíduos LGBTT nos municípios de Juazeiro do Norte e Crato, Ceará, Brasil. Evidenciou-se prevalência de violência psicológica, sendo insultos por parte dos agressores localizados longe da vítima o tipo mais comum. Os principais agressores são pessoas desconhecidas e as reações físicas, como agressões, são as principais formas de reação. Os índices de violência psicológica cometidas contra essa população são elevados; achado importante para o planejamento de estratégias para redução desse agravo.

PALAVRAS-CHAVE Comportamento sexual; Violência; Homossexualidade; Bissexualidade.

ABSTRACT This study aimed to determine the profile of psychological violence against Lesbians, Gays, Bisexuals, Transvestites and Transgender (LGBTT) in Ceará. Quantitative study arried out with 316 LGBTT in individuals in the municipalities of Juazeiro do Norte and Crato, Ceará, Brazil. It showed a prevalence of psychological violence, and insults by the attackers located away from the victim were the most common type. The main perpetrators are unknown people and physical reactions, such as aggression, the main forms of reaction. The rates of psychological violence committed against this population are high; found important for planning strategies for the reduction of this aggravation.

KEYWORDS Sexual behavior; Violence; Homosexuality; Bisexuality. 


\section{Introdução}

Os aspectos sexuais inerentes aos seres humanos vêm despertando o interesse de inúmeras pesquisas científicas em diferentes momentos históricos e distintas áreas do conhecimento. Nos dias atuais, esse interesse parece se direcionar para o estudo das manifestações preconceituosas e discriminatórias contra indivíduos que assumem uma identidade de gênero e orientação sexual adversa aos padrões heteronormativos hegemônicos vigentes, como a população de Lésbicas, Gays, Bissexuais, Travestis e Transexuais (LGBTT) (RAMOS, CARRARA, 2006).

A heteronormatividade constitui-se em um sistema de valores e ideologias sociais e culturais, composto por indivíduos heterossexuais, que consideram a heterossexualidade a única orientação sexual natural (AlbuQUerQue et Al., 2013). Argumentos reforçam tal condição, visto que características biológicas dos corpos masculinos e femininos consideradas naturais, por fazerem parte da natureza humana, são utilizadas para direcionar diferenças comportamentais, intelectuais, de habilidades e estilos de vida de homens e mulheres, entre essas, está inclusa a atração afetivo - sexual por pessoas do sexo oposto - a heterossexualidade (FISCHER, 2005). Assim, qualquer orientação sexual contrária a esses padrões, como a homossexualidade e bissexualidade, por serem contrárias à dita 'naturalidade imposta', não poderiam ser aceitas socialmente.

Nesse sentido, a comunidade LGBTT, ao fugir do padrão heteronormativo vigente, provoca conflitos ao contrariar um modelo hegemônico e um sistema de valores, condutas e padrões sociais e sexuais (ALBUQUerQUe ET AL., 2013).

Dessa forma, qualquer manifestação sexual contrária aos padrões heteronormativos pode vir a ser alvo de violência física, sexual e/ou psicológica. Ramos e Carrara (2006) destacam a crescente mobilização LGBTT, que busca dar visibilidade aos crimes motivados pela orientação sexual não heterossexual, divulgando o termo 'homofobia' para designar tais atos violentos contra toda a comunidade LGBTT. Atualmente, variantes como LGBTTfobia são adotadas, porque incluem de forma mais explícita lésbicas, gays, bissexuais, travestis e transexuais. Leony (2006) caracteriza a homofobia como o ódio explícito, persistente e generalizado, manifestando-se em uma escala de violência que varia desde as agressões verbais contra a honra e a moral, até os episódios de violência física, inclusive letais, consumados com requintes de crueldade.

Segundo dados da Secretaria de Direitos Humanos da Presidência da República (SDH/PR), no ano de 2012, por meio do Disque 100 , foram registradas 27,34 violações de direitos humanos por dia contra o grupo LGBTT, de caráter homofóbico, o que caracteriza um aumento de $46,6 \%$ das violações em relação ao ano de 2011. Conforme o relatório, a principal manifestação de violência perpetrada contra a população LGBTT é a psicológica, perfazendo um total de 83,2\% contra 42,5\% em 2011 (BRASIL, 2013).

Várias podem ser as manifestações de violência psicológica dirigida à população LGBTT, tais como ameaças; humilhações; chantagens; cobranças para mudança de comportamento; discriminação; exploração; crítica pelo desempenho sexual e proibição de socialização, provocando, assim, o isolamento dos amigos e familiares (BRASIL, 2002). De fato, considera-se como violência psicológica agressões verbais ou gestuais com o objetivo de aterrorizar, rejeitar, humilhar a vítima, restringir sua liberdade ou, ainda, isolá-la do convívio social.

Dessa forma, considera-se a violência perpetrada contra LGBTT um importante estressor social que resulta em impactos negativos na saúde mental e qualidade de vida de LGBTT, incluindo um aumento de quase seis vezes para ocorrência de quadros depressivos (RYAN ET AL., 2009) e seus desdobramentos, como os sentimentos de culpa, 
medo, desconfiança, confusão, insegurança, ansiedade, vergonha, isolamento social, dificuldades de estabelecer e manter relacionamentos amorosos, disfunções sexuais, hostilidade, distúrbios alimentares e uso/ abuso de substâncias psicoativas (CARDOso, FERRO, 2012).

Assim, considerando os altos índices de violência psicológica cometida contra a população LGBTT e suas consequências, objetivou-se determinar o perfil da violência psicológica/verbal perpetrada contra esta população na Região do Cariri, interior do Ceará, em face da inexistência de estudos acerca dessa temática em LGBTT no interior do Nordeste.

A realização deste estudo é de importante relevância científica e social, pois poderá proporcionar direcionamentos para o desenvolvimento de ações e políticas governamentais que assegurem os direitos da população LGBTT no efetivo exercício da cidadania.

\section{Método}

Trata-se de um estudo transversal, de abordagem quantitativa, em que para recrutamento dos participantes realizou-se uma abordagem intencional de LGBTT e definição da amostra por conveniência. Os dados foram coletados durante movimentos reivindicatórios da categoria LGBTT, intitulados 'Parada Gay', promovidos em junho de 2013, nos municípios de Juazeiro do Norte e de Crato, ambos localizados na região sul do estado do Ceará, Brasil.

Foram abordados intencionalmente 400 indivíduos. Destes, 30 foram excluídos por serem simpatizantes e/ou curiosos e 54 que se recusaram a participar da pesquisa, perfazendo uma amostra final de 316 LGBTT que consentiram participação.

O processo de coleta de dados ocorreu por meio de um conjunto de questões estruturadas, previamente formuladas pela equipe pesquisadora, acerca da: I) vitimização por violência psicológica e sua tipificação, II) orientação sexual dos agressores e seu grau de relacionamento com a vítima e II) formas de reação contra a violência psicológica sofrida e problemas decorrentes dos atos violentos sofridos. Para cada pergunta, eram esperadas respostas tipo SIM ou NÃO.

As informações foram coletadas por equipe previamente treinada, em local reservado, com duração média aproximada de 15 minutos. Ressalta-se que foram resguardados a participação voluntária, segurança e o bem-estar dos participantes recrutados, mediante a adoção do Termo de Consentimento Livre e Esclarecido (TCLE), respeitando-se durante o desenvolvimento do estudo a autonomia, a dignidade e o anonimato dos participantes.

Após o término das entrevistas, os participantes foram identificados com adesivos confeccionados com o texto 'Eu participei da pesquisa', como estratégia para diminuir o viés de seleção, ao evitar que o mesmo indivíduo fosse abordado e participasse novamente.

Os dados foram agrupados por frequência de aparecimento das respostas e apresentados sob a forma de tabelas. Os resultados foram apresentados mediante frequência absoluta (fa) e frequência relativa (fr), esta última sendo representada em valores percentuais.

A etapa da discussão dos dados ocorreu em conformidade com a literatura pertinente, sendo a análise dos dados realizada por meio da comparação dos resultados encontrados com outras investigações que abordassem aspectos relativos à violência perpetrada contra a população LGBTT, no intuito de identificar possíveis evidências consensuais e divergências entre as investigações.

Esse estudo constitui fragmento de uma pesquisa ampliada intitulada 'A saúde LGBTT: LGBTTfobia, violência, drogas 
e itinerários terapêuticos na busca pela igualdade de direitos e exercício da cidadania', aprovado pelo Comitê de Ética em Pesquisa da Faculdade de Medicina do ABC, com número de parecer 346.679, obedecendo aos princípios da Resolução no 466/12, do Conselho Nacional de Saúde, que dispõe sobre as diretrizes e normas para realização de pesquisas envolvendo seres humanos.

\section{Resultados}

Participaram do estudo 316 LGBTT, predominantemente do sexo masculino $(n=223$, $70,6 \%$,), gays ( $n=162,51,3 \%)$, com identidade de gênero masculina ( $n=200,63,3 \%)$, orientação sexual homossexual $(n=253,78,7 \%)$ e que em sua maioria $(n=249)$ já sofreram violência psicológica/verbal alguma vez na vida (tabela 1).

Tabela 1. Perfil sociodemográfico e violência psicológica sofrida por LGBTT. Juazeiro do Norte e Crato, Ceará, Brasil, junho de 2013

\begin{tabular}{|c|c|c|c|}
\hline Característica & $\mathrm{fa}$ & $\operatorname{fr}(\%)$ & № Vítimas Violência \\
\hline \multicolumn{4}{|l|}{ Sexo biológica } \\
\hline Masculino & 223 & 70,6 & 176 \\
\hline Feminino & 93 & 29,4 & 73 \\
\hline \multicolumn{4}{|l|}{ Identidade de gênero } \\
\hline Masculino & 200 & 63,3 & 158 \\
\hline Feminino & 113 & 35,8 & 89 \\
\hline Ambas (Masculino e Feminino) & 03 & 0,9 & 02 \\
\hline \multicolumn{4}{|l|}{ Orientação sexual } \\
\hline Homossexual & 253 & 78,7 & 208 \\
\hline Bissexual & 63 & 21,3 & 41 \\
\hline \multicolumn{4}{|l|}{ Autodefinição } \\
\hline Gays & 162 & 51,3 & 127 \\
\hline Lésbicas & 71 & 22,5 & 56 \\
\hline Bissexuais & 63 & 19,9 & 50 \\
\hline Travestis & 14 & 4,4 & 11 \\
\hline Transexuais & 06 & 1,9 & 05 \\
\hline \multicolumn{4}{|l|}{ Cor/etnia } \\
\hline Amarelo & 02 & 0,6 & 02 \\
\hline Branca & 87 & 27,5 & 68 \\
\hline Parda & 196 & 62,1 & 155 \\
\hline Negro & 31 & 9,8 & 24 \\
\hline
\end{tabular}




\begin{tabular}{|c|c|c|c|}
\hline \multicolumn{4}{|l|}{ Escolaridade } \\
\hline Ensino fundamental completo & 25 & 7,9 & 20 \\
\hline Ensino fundamental incompleto & 25 & 7,9 & 20 \\
\hline Ensino médio completo & 108 & 34,2 & 85 \\
\hline Ensino médio iccompleto & 72 & 22,8 & 57 \\
\hline Ensino superior completo & 27 & 8,5 & 21 \\
\hline Ensino superior incompleto & 45 & 14,3 & 35 \\
\hline Pós-graduação & 14 & 4,4 & 11 \\
\hline \multicolumn{4}{|l|}{ Estado civil } \\
\hline Solteiro & 200 & 63,3 & 158 \\
\hline Namorando & 72 & 22,8 & 57 \\
\hline União estável & 29 & 9,2 & 23 \\
\hline Casado/a & 12 & 3,8 & 09 \\
\hline Divorciado/a & 01 & 0,3 & 01 \\
\hline Viúvo & 02 & 0,6 & 01 \\
\hline
\end{tabular}

Fonte: Elaboração própria.

Entre os principais tipos de violência psicológica comumente perpetradas contra a população LGBTT, estão os insultos proferidos por indivíduos que se encontram longe (a certa distância física do LGBTT) (n=193,
77,5\%), seguidos de insultos proferidos por indivíduos que estão perto (distância física reduzida) ( $\mathrm{n}=186,74,7 \%)$ e, posteriormente por pressões para alterar a orientação sexual $(\mathrm{n}=167,67,1 \%)$ (tabela 2$)$.

Tabela 2. Violência psicológica por subtipo em LGBTT. Juazeiro do Norte e Crato, Ceará, Brasil, junho de 2013

\begin{tabular}{lcccccr}
\hline Variáveis dicotômicas & \multicolumn{2}{c}{ Sim } & \multicolumn{2}{c}{ Não } & \multicolumn{2}{c}{ N/R } \\
\hline Tipo de violência e frequência & $\mathrm{fa}$ & $\mathrm{fr}(\%)$ & $\mathrm{fa}$ & $\mathrm{fr}(\%)$ & $\mathrm{fa}$ & $\mathrm{fr}(\%)$ \\
\hline Insultos de longe & 193 & 77,5 & 48 & 19,3 & 08 & 3,2 \\
Insultos de perto & 186 & 74,7 & 53 & 21,2 & 10 & 4,1 \\
Ameaça de morte & 56 & 22,5 & 173 & 69,5 & 20 & 8,0 \\
Ameaças de fazer algum mal & 94 & 37,8 & 138 & 55,3 & 17 & 6,9 \\
Agravos ao patrimônio & 42 & 16,9 & 183 & 73,5 & 24 & 9,6 \\
Ameaças aos familiares & 67 & 27 & 163 & 65,4 & 19 & 7,6 \\
Ameaça de revelar a orientação sexual & 83 & 33,3 & 144 & 57,9 & 22 & 8,8 \\
\hline
\end{tabular}




\begin{tabular}{lcccccc}
\hline Tabela 2 (cont.) & \multicolumn{7}{c}{} & & & \\
\hline Perseguição & 94 & 37,8 & 139 & 55,8 & 16 & 6,4 \\
Gritos de intimidação & 140 & 56,3 & 92 & 36,8 & 17 & 6,9 \\
Retenção de economias & 41 & 16,5 & 183 & 73,5 & 25 & 10,0 \\
Cárcere privado & 73 & 29,3 & 160 & 64,3 & 16 & 6,4 \\
Evitar contado com outras pessoas & 90 & 36,2 & 143 & 57,5 & 16 & 6,3 \\
Pressão para mudar a orientação sexual & 167 & 67,1 & 78 & 31,3 & 04 & 1,6 \\
\hline
\end{tabular}

Fonte: Elaboração própria.

A descrição do tipo de relacionamento entre vítimas e agressores evidenciou, conforme tabela 3, a predominância de agressores conhecidos, com destaque para membros familiares ( $n=112,45,0 \%)$, enquanto diante dos agressores desconhecidos, sobressaíram-se as pessoas da rua
( $\mathrm{n}=113,45,4 \%$ ). Quanto ao grau de envolvimento entre vítimas e agressores de modo geral, sobressaíram-se as pessoas desconhecidas na rua $(\mathrm{n}=113,45,4 \%)$, seguido dos conhecidos familiares $(n=112,45,0 \%)$ e amigos $(n=99,39,8 \%)$ respectivamente (tabela 3).

Tabela 3. Grau de envolvimento e/ou forma de relacionamento da vítima com os principais agressores. Juazeiro do Norte e Crato, Ceará, Brasil, junho de 2013

\begin{tabular}{llcrrr}
\hline Variáveis dicotômicas & & \multicolumn{2}{c}{ Sim } & \multicolumn{2}{c}{ Não } \\
\hline Envolvidos e frequência & & $\mathrm{fa}$ & $\mathrm{fr}(\%)$ & $\mathrm{fa}$ & $\mathrm{fr}(\%)$ \\
\hline & Companheiro & 12 & 4,8 & 237 & 95,2 \\
& Ex-companheiro & 18 & 7,2 & 231 & 92,8 \\
& Familiar & 112 & 45,0 & 137 & 55,0 \\
& Amigo & 99 & 39,8 & 150 & 60,2 \\
& Parceiro ocasional & 12 & 4,8 & 237 & 95,2 \\
\hline \multirow{2}{*}{ Desconhecido } & Profissional de saúde & 3 & 1,2 & 246 & 98,8 \\
& Profissional de educação & 12 & 4,8 & 237 & 95,2 \\
& Profissional de justiça & 15 & 6,0 & 234 & 94,0 \\
\hline Outros & Pessoas na rua & 113 & 45,4 & 136 & 54,6 \\
\hline
\end{tabular}

Fonte: Elaboração própria.

Quanto às formas de reação aos atos de violência psicológica sofrida, evidenciou-se que, conforme tabela 4, reações físicas, caracterizadas como agressões ( $\mathrm{n}=137,55 \%)$, constituíram-se a principal forma de reação de LGBTT perante a violência sofrida. Em segundo lugar, tem-se a procura por amigos ( $\mathrm{n}=133,53,4 \%)$, o choro ( $n=120,48,1 \%)$, a ingestão de bebida alcoólica $(n=80,32,0 \%)$ e a procura por familiares $(\mathrm{n}=65,26,1 \%)$ (tabela 4$)$. 
Tabela 4. Formas de reação à violência psicológica sofrida por LGBTT. Juazeiro do Norte e Crato, Ceará, Brasil, junho de 2013

\begin{tabular}{|c|c|c|c|c|c|c|}
\hline \multirow{2}{*}{$\begin{array}{l}\text { Variáveis dicotômicas } \\
\text { Tipo de violência e frequência }\end{array}$} & \multicolumn{2}{|c|}{ Sim } & \multicolumn{2}{|c|}{ Não } & \multicolumn{2}{|c|}{$N / R$} \\
\hline & fa & $\mathrm{fr}(\%)$ & $\mathrm{fa}$ & $\operatorname{fr}(\%)$ & $\mathrm{fa}$ & $\operatorname{fr}(\%)$ \\
\hline Reagiu fisicamente (agressões) & 137 & 55,0 & 100 & 40,2 & 12 & 4,8 \\
\hline Reagiu verbalmente & 52 & 20,9 & 181 & 72,7 & 16 & 6,4 \\
\hline Mentiu & 62 & 24,9 & 171 & 68,7 & 16 & 6,4 \\
\hline Procurou ajuda na igreja & 30 & 12,0 & 205 & 82,3 & 14 & 5,7 \\
\hline Procurou ajuda em centro psicológico & 33 & 13,2 & 203 & 81,5 & 13 & 5,3 \\
\hline Procurou cuidados em saúde & 50 & 20 & 185 & 74,3 & 14 & 5,7 \\
\hline Sentiu-se culpado & 53 & 21,3 & 178 & 71,5 & 18 & 7,2 \\
\hline Ingeriu drogas & 20 & 8,1 & 211 & 84,7 & 18 & 7,2 \\
\hline Ingeriu bebidas alcoólicas & 80 & 32,0 & 155 & 62,3 & 14 & 5,7 \\
\hline Procurou ajuda na delegacia & 40 & 16,0 & 194 & 77,9 & 15 & 6,1 \\
\hline Procurou ajuda de familiares & 65 & 26,1 & 170 & 68,2 & 14 & 5,7 \\
\hline Procurou ajuda de amigos & 133 & 53,4 & 104 & 41,8 & 12 & 4,8 \\
\hline Escondeu-se & 60 & 24,1 & 171 & 68,7 & 18 & 7,2 \\
\hline Mudou de endereço & 21 & 8,4 & 210 & 84,4 & 18 & 7,2 \\
\hline Chorou & 120 & 48,1 & 116 & 46,6 & 13 & 5,3 \\
\hline Não apresentou reação & 51 & 20,5 & 173 & 69,5 & 25 & 10,0 \\
\hline
\end{tabular}

Fonte: Elaboração própria.

Ante os problemas psicológicos associados à violência sofrida, conforme tabela 5 , predominou o sentimento de tristeza
( $\mathrm{n}=130,52,2 \%)$, seguido por baixa autoestima ( $\mathrm{n}=94,37,7 \%)$ e ansiedade $(\mathrm{n}=89,35,7 \%)$ (tabela 5).

Tabela 5. Problemas psicológicos associados à violência sofrida em LGBTT. Juazeiro do Norte e Crato, Ceará, Brasil, junho de 2013

\begin{tabular}{lcrrr}
\hline Variáveis dicotômicas & \multicolumn{2}{c}{ Sim } & \multicolumn{2}{c}{ Não } \\
\hline Problemas psicológicos e frequência & $\mathrm{fa}$ & $\mathrm{fr}(\%)$ & $\mathrm{fa}$ & $\mathrm{fr}(\%)$ \\
\hline Depressão & 70 & 28,1 & 179 & 71,9 \\
Ansiedade & 89 & 35,7 & 160 & 64,3 \\
Pânico & 33 & 13,3 & 216 & 86,7 \\
Insônia & 70 & 28,1 & 179 & 71,9 \\
Dificuldade de concentração & 48 & 19,3 & 201 & 80,7 \\
\hline
\end{tabular}




\begin{tabular}{lcccc}
\hline Tabela 5 (cont.) & & & & \\
\hline Pensamento suicida & 50 & 20,1 & 199 & 79,9 \\
Tristeza & 130 & 52,2 & 119 & 47,8 \\
Isolamento social & 53 & 21,3 & 196 & 78,7 \\
Irritabilidade & 71 & 28,5 & 178 & 71,5 \\
Baixa autoestima & 94 & 37,7 & 155 & 62,3 \\
\hline
\end{tabular}

Fonte: Elaboração própria.

\section{Discussão}

Os resultados do estudo indicam prevalência de violência psicológica em 249 LGBTT $(78,8 \%)$ e corroboram o levantamento da $\mathrm{SDH} / \mathrm{PR}$ realizado em 2012 por meio da contabilização do quantitativo de denúncias realizadas ao Disque 100, em que foram registradas 9.982 violações, apontando a violência psicológica como a principal manifestação de violência perpetrada contra a população LGBTT $(83,2 \%)$, seguida de discriminação (74,01\%), violência física $(32,68 \%)$ e violência sexual (4,18\%) (BRASIL, 2013).

Pelullo, Giuseppe e Angelino (2013), em estudo realizado com mil lésbicas, gays e bissexuais na cidade de Nápoles, na Itália, entrevistas face a face, identificou que 28,3\% dos participantes relataram que tinham sofrido pelo menos um episódio de vitimização em sua vida, entre esses, a prevalência de assédio verbal foi a mais frequente, totalizando $85,2 \%$.

Esses achados apontam a violência psicológica como a principal manifestação de violência cometida contra LGBTT. Ademais, esse tipo de agravo constitui a forma mais difícil de ser visibilizada, uma vez que se apoia em mecanismos simbólicos de poder que estruturam a sociedade, fazendo com que as vítimas não a percebam como violência, pois preconceito e LGBTTfobia são caracterizados como condições naturalizadas na sociedade brasileira (BRASIL, 2012).

Considerando a predominância de indivíduos do sexo masculino vítimas de abusos emocionais ( $n=176)$, observa-se que esse achado coincide com dados da SDH/PR (BRASIL, 2013), em que a violência psicológica foi a mais reportada por indivíduos desse sexo (71,38\%). Ainda, os dados obtidos corroboram os resultados de estudo conduzido por Pelullo, Giuseppe e Angelino (2013), no qual a violência psicológica esteve presente em $60,2 \%$ dos pesquisados do sexo masculino.

Esse fato, agregado à maior vitimização de indivíduos com orientação sexual homossexual, seria um indicativo da manifestação do poder machista e heteronormativo, que hierarquiza e inferioriza mulheres e homossexuais (PINO, 2007).

Uma possível explicação para esse fenômeno encontra-se no fato de que indivíduos gays, por romperem com ideários de superioridade, força e virilidade como atributos masculinos, acabam se tornando alvos de violência e discriminação social por fugirem dos papéis sexuais e sociais culturalmente atribuídos aos homens.

A existência de pessoas negras como vítimas de violência psicológica neste estudo ( $\mathrm{n}=31,9,8 \%)$ também coincide com outros estudos. Os dados do SDH/PR de 2012 revelaram que LGBTT sofreram com discriminação racial/étnica (40,55\%) (BRASIL, 2013; WAISELFISZ, 2014).

Em relação aos principais tipos de violência psicológica, os resultados encontrados nesta pesquisa divergem das informações apresentadas no relatório de 
violência homofóbica no Brasil pela $\mathrm{SDH} /$ PR, que apontou humilhações (35,32\%), hostilizações (32,27\%) e ameaças (15,78\%) como os subtipos mais frequentes desse tipo de violência (BRASIL, 2013). No entanto, considerando que os insultos sejam uma forma de humilhação e que os gritos, de hostilização, os resultados dessas investigações se assemelham.

Quando se associa os dados da violência psicológica às formas de relacionamento e/ou envolvimento das vítimas, os dados encontrados condizem com os apresentados pela SDH/PR no ano de 2011. Os dados desse ano revelam que foram denunciadas por intermédio do Disque 1006.809 violações dos direitos humanos, apontando que os principais agressores são pessoas conhecidas $(61,9 \%)$ em relação aos desconhecidos (29,4\%). Ademais, esse levantamento aponta os familiares $(38,02 \%)$, vizinhos $(35,8 \%)$ e companheiros (11,6\%) como principais agressores entre aqueles que são conhecidos, assemelhando-se aos resultados encontrados neste estudo (BRASIL, 2012).

Diante desse cenário, em revisão sistemática conduzida por Finneran e Stepherson (2013) sobre Violência por Parceiro Íntimo (VPI) entre homens norte-americanos que fazem sexo com homens (HSH), em que, de um total de 576 estudos revisados, 28 preencheram critérios de inclusão, revelou-se o crescimento em larga escala de pesquisas relacionadas com a violência por parceiro íntimo entre HSH. Com base nos resultados da revisão, foi identificada prevalência de violência entre parceiros íntimos que variou de $29,7 \%$ a $78 \%$ A violência psicológica, por sua vez, entre esses indivíduos, foi caracterizada como menos frequente em relação aos demais tipos de violência, com sua escala variando entre $5,4 \%$ e $73,2 \%$.

Considerando as formas de reação das vítimas diante da violência sofrida, constatou-se que as reações físicas, como as agressões, constituíram-se a principal forma de reação de LGBTT vitimizados (55\%). As reações físicas enquanto manifestações de enfretamento à violência podem ser explicadas a partir da perspectiva teórica da aprendizagem social (BANDURA, 1973), a qual argumenta que um indivíduo que vivencia violência, encontra no ato em si o aval que o legitima a reagir/revidar, podendo utilizar também a violência como alternativa para tentar se impor e resolver seus conflitos. Além disso, infere-se que a carência de ações protetivas do Estado perante esse grupo constitui-se como potencial agravante das violências perpetradas.

A procura por amigos, como a segunda forma de reação à violência, sugere que essa seja uma alternativa para encontrar amparo na rede de relações sociais, como uma estratégia de enfretamento dos problemas, reforçando, dessa maneira, a importância do apoio social e familiar. Nessa perspectiva, a procura por amigos, sobrepondo-se à busca pela ajuda de familiares, põe em cheque a atuação do núcleo familiar no suporte aos indivíduos LGBTT.

Muitas vezes, a busca de LGBTT pelo apoio da família não acontece, visto que são poucas as famílias que aceitam e convivem bem com essa população, estando mais presente a intolerância e o inconformismo (PALMA; LEVANDOWSKI, 2008). Segundo os mesmos autores, quando essa população se sente amada e protegida por seus familiares, os problemas sociais decorrentes da orientação sexual homossexual e bissexual acabam se tornando menos difíceis de enfrentar.

A falta de apoio familiar e social gera impacto negativo sobre o estado emocional de indivíduos LGBTT, que passam a relatar altos níveis de humor deprimido e níveis elevados de LGBTTfobia internalizada (NYAMETHI ET AL., 2012). De fato, diversos sentimentos associam-se aos atos de violência e estes mantêm relação com as estratégias de enfrentamento e suporte sociais disponíveis às vítimas, impactando em agravos à saúde, detidamente por meio da manifestação de desordens mentais, conforme achados constatados no 
presente estudo, no qual predominaram sentimentos de tristeza $(52,2 \%)$, baixa autoestima (37,7\%) e ansiedade (35,7\%).

Mustanski et al. (2014) discorre sobre os problemas psicossociais em grupos marginalizados, entre os quais se destacam os homossexuais. Suas pesquisas apontam as sindemias como uma explicação teórica para as disparidades em saúde dessa população. Segundo esses autores, a sindemia ocorre quando várias epidemias e fatores de risco interagem entre si, agravando o risco e as consequências das doenças, sobretudo em indivíduos que são confrontados com as adversidades da vida, principalmente manifestadas sob as formas de marginalização social e vitimização, tornando-os suscetíveis ao desenvolvimento de problemas de saúde psicossociais, tais como depressão, baixa autoestima e uso de substâncias, aumentando, dessa forma, o risco do indivíduo cometer suicídio (MUSTANSKI ET AL., 2014).

É fato que LGBTT experimentam níveis mais elevados de sintomas depressivos quando comparados a homólogos heterossexuais (HATZENBUEHLER, MCLAUGHLIN, NOLENHOEKSEMA, 2008). Sintomatologia depressiva em LGBTT está associada às inúmeras consequências negativas na saúde, incluindo experimentação, uso e ou abuso de drogas e tentativas de suicídio (KING ET AL., 2008). Assim, as evidências indicam que o sofrimento psíquico eleva a vulnerabilidade para adoção de comportamentos de risco nesse grupo.

Estudo realizado com 7.403 pessoas entrevistadas na Inglaterra sobre prevalência de resultados de saúde mental por orientação sexual, demonstrou que desfechos psicológicos negativos foram significativamente mais prevalentes em pessoas que se autoidentificaram como não heterossexuais, com razão de chance de desenvolver transtorno neurótico geral $(\mathrm{OR}=1,47)$, episódio depressivo maior $(\mathrm{OR}=1,80)$, psicose $(\mathrm{OR}=3,75)$, dependência de drogas no ano anterior $(\mathrm{OR}=$ 1,70 ), dependência de álcool nos últimos seis meses $(\mathrm{OR}=2,05)$, pensamentos suicidas
$(\mathrm{OR}=1,85)$, tentativas de suicídio $(\mathrm{OR}=2,21)$ e automutilação ( $\mathrm{OR}=2,82)$ (CHAKRABORTY, 2011).

Esses resultados reforçam a importância de uma maior atenção à saúde voltada para as necessidades e especificidades da população LGBTT vítima de violência psicológica. Dessa forma, cabe aos profissionais de saúde e de segurança pública identificar os indivíduos vítimas desse tipo de violência a fim de atuar no desenvolvimento de estratégias voltadas para a redução de danos e complicações, prevenção de riscos e promoção à saúde desse público.

\section{Considerações finais}

Os achados do estudo permitiram caracterizar a violência psicológica como um fenômeno complexo e multidimensional, enquanto reflexo do preconceito e hostilidade direcionados às expressões de sexualidade não heteronormativas, além de figurar como problema de saúde pública.

Ademais, a naturalização dos atos de violência contra LGBTT e a omissão social permissiva a esses atos atuam como fatores que perpetuam e disseminam um tipo de violência não tão aparente como as agressões físicas, mas uma violência silenciosa e muitas vezes imperceptível, que produz consequências graves, podendo levar suas vítimas a desenvolverem problemas de ordem mental e, em muitos casos, ao suicídio.

No que concerne às limitações da pesquisa, o delineamento metodológico transversal impossibilitou estabelecer uma associação causal entre a violência psicológica e as variáveis estudadas. Entre os pontos fortes desse estudo, destaca-se a diversidade de variáveis apresentadas e a inovação metodológica que, segundo o conhecimento de seus autores, constitui o primeiro estudo face a face na região do Cariri a estudar violência psicológica contra a população LGBTT.

Almeja-se, ao visibilizar a violência psicológica/verbal enquanto problema que afeta a 
saúde da população LGBTT, contribuir com informações que possam nortear a construção de ações e intervenções no campo de políticas públicas voltadas ao combate da LGBTTfobia e suas consequências, assim como suprir a carência de dados científicos regionais sobre o assunto, inserindo as discussões no âmbito nacional e internacional e estimulando a produção de novos estudos sobre a temática.

\section{Referências}

ALBUQUERQUE, G. A. et al. Homossexualidade e o direito à saúde: um desafio para as políticas públicas de saúde no Brasil. Saúde em Debate, Rio de Janeiro, v. 37, n. 98, p. 516-524, jul./set. 2013.

BANDURA, A. Aggression: a social learning analysis. Englewood Cliffs: Prentice-Hall, 1973.

BRASIL. Ministério da Saúde. Secretaria de Políticas de Saúde. Violência intrafamiliar: orientações para a prática em serviço. Brasília, DF, 2002. (Cadernos de Atenção Básica, 8; Série A - Normas e Manuais Técnicos, 131). Disponível em: <http://bvsms.saude. gov.br/bvs/publicacoes/cd05_19.pdf>. Acesso em: 21 mar. 2016.

Secretaria de Direitos Humanos. Relatório sobre violência homofóbica no Brasil: ano de 2011. Brasília, DF, 2012. Disponível em: <http://www. sdh.gov.br/assuntos/lgbt/pdf/relatorio-violenciahomofobica-2011-1>. Acesso em: 21 mar. 2016.

Secretaria de Direitos Humanos. Relatório sobre violência homofóbica no Brasil: ano de 2012. Brasília, DF, 2013. Disponível em: <http://www. sdh.gov.br/assuntos/lgbt/pdf/relatorio-violenciahomofobica-2011-1>. Acesso em: 21 mar. 2016.
CARDOSO, M. R.; FERRO, L. F. Saúde e população LGBTT: demandas e especificidades em questão. Psicologia: Ciência e Profissão, Brasília, DF, v. 32, n. 3, 552-63, 2012.

CHAKRABORTY, A. et al. Mental health of the nonheterosexual population of England. Br J Psychiatry, London, v. 198, n. 2, p. 143-48, jan. 2011.

FINNERAN, C.; STEPHENSON, R. Intimate Partner Violence among Men Who Have Sex with Men: A Systematic Review. Trauma, violence \& abuse, Thousand Oaks, v. 14, n. 12, p. 168-85, 2013.

FISCHER, R. M. B. Mídia e juventude: experiências do público e do privado na cultura. Cad. Cedes, Campinas, v. 25 , n. 65 , p. $43-58,2005$.

HATZENBUEHLER, M. L.; MCLAUGHLIN, K. A.; NOLEN-HOEKSEMA, S. Emotion regulation and internalizing symptoms in a longitudinal study of sexual minority and heterosexual adolescents. J Child Psychol Psychiatry, Oxford, v. 49, n. 12, p. 1270-78, dez. 2008.

KING, M. et al. A systematic review of mental disorder, suicide and deliberate self-harm in lesbian, gay and bisexual people. BMC Psychiatry, London, v. 8, n. 70, p. 1333-45, 2008. 
LEONY, M. C. Homofobia, controle social e política pública de atendimento. 2006. 116 f. Monografia (Pósgraduação Lato Sensu em Gestão Estratégica em Segurança Pública). Universidade Federal de Sergipe, Aracaju, 2006.

MUSTANSKI, B. et al. Syndemic of psychosocial health disparities and associations with risk for attempting suicide among young sexual minority men. American Journal of Public Health, New York, v. 104, n. 2, p. 28794, 2014

NYAMATHI, A. et al. Correlates of depressed mood among young stimulant-using homeless gay and bisexual men. Issues Ment Health Nurs, London, v. 33, n. 10, p. 641-49, out. 2012.

PALMA, Y. A.; LEVANDOWSKI, D. C. Vivências pessoais e familiares de homossexuais femininas. Psicol. estud., Maringá, v. 13, n. 4, p. 771-79, dez. 2013

PELULLO, C. P.; GIUSEPPE, G. D.; ANGELILLO, I. F. Frequency of discrimination, harassment, and violence in lesbian, gay men, and bisexual in Italy. Plos One, San Francisco, v. 8, n. 8, p. 1284-95, 2013.
PINO, N. P. A teoria queer e os intersex: experiências invisíveis de corpos des-feitos. Cadernos Pagu [Internet], n. 28, p. 149-74, 2007. Disponível em: $<$ http://www.scielo.br/pdf/cpa/n28/08.pdf >. Acesso em: 21 mar. 2016.

RAMOS, S.; CARRARA, S. A constituição da problemática da violência contra homossexuais: a articulação entre ativismo e academia na elaboração de políticas públicas. Physis, Rio de Janeiro, v. 16, n. 2, p. 185-205, 2006.

RYAN, C. et al. Family rejection as a predictor of negative health outcomes in white and latino lesbian, gay, and bisexual young adults. Pediatrics, Springfield, v. 123, n. 1, 346-52, jan. 2009.

WAISELFISZ, J. J. Mapa da violência 2014: Homocídios e juventude no Brasil. [Internet]. Brasília, DF, 2014. Disponível em: <http://www.mapadaviolencia.org.br/ pdf2014/Mapa2014_JovensBrasil_Preliminar.pdf >. Acesso em: 4 abr. 2016.

Recebido para publicação em novembro de 2015

Versão final em março de 2016

Conflito de interesses: inexistente

Suporte financeiro: não houve 